\title{
AVIFAUNA DEL ESTERO DE PUNTA BANDA, BAJA CALIFORNIA, MÉXICO
}

\author{
Luis C. JiMÉnez PÉREZ1* ${ }^{*}$, Horacio de la Cueva², \\ Fernando MOLINA-PERALTA ${ }^{3}$ y Arnulfo ESTRADA-RAMírez 3
}

1 *Universidad de Guadalajara. Centro Universitario de la Costa. Av. Universidad de Guadalajara No. 203 Delegación Ixtapa. Puerto Vallarta, Jalisco, MÉXICO. C.P. 48280. 1jimenez@pv.udg.mx

${ }^{2}$ Centro de Investigación Científica y Educación Superior de Ensenada.

Departamento de Biología de la Conservación. Km 107 Carretera Tijuana-Ensenada, Apdo. Postal 360, C. P. 22830 Ensenada, Baja California, MÉXICO. cuevas@ cicese.mx

${ }^{3}$ Secretaría de Marina. Dirección de Investigación y Desarrollo.

Estación de Investigación Oceanográfica de Ensenada. Ave. Agustín Sanginés y Boulevard Costero s/n. Ensenada, Baja California, MÉXICO.

Jiménez-Pérez, L. C., H. de la Cueva, R. F. Molina-Peralta y A. Estrada-Ramírez. 2009. Avifauna del Estero de Punta Banda, Baja California, México. Acta Zoológica Mexicana (n. s.), 25(3): 589-608.

RESUMEN. Entre marzo de 2004 y marzo de 2005 se realizaron veinticuatro salidas de campo quincenales de avistamiento y registro de aves costeras en 6 unidades ambientales del Estero de Punta Banda, Baja California, México. Se registraron 35 familias y 104 especies; 64 estuvieron asociadas al ambiente acuático. La estructura de las comunidades de aves acuáticas y terrestres presentó una secuencia temporal que se caracterizó por la formación de dos grupos asociados con las condiciones de invierno o verano y otro más pequeño formado por los censos efectuados en la transición entre ambas estaciones. Doce especies aparecen enlistadas en alguna categoría de protección en la Norma Oficial Mexicana. Se registró el éxito reproductivo de dos especies residentes (Rallus longirostris e Himantopus mexicanus) en áreas disturbadas por estructuras costeras hechas por el hombre. Los resultados indican que el Estero de Punta Banda es importante como área de reproducción, alimentación y descanso de muchas especies de aves residentes y migratorias. Sin embargo, el acelerado crecimiento urbano de la ciudad de Ensenada, la contaminación y el uso desordenado de sus recursos amenaza su presencia y supervivencia.

Palabras clave: Biodiversidad, avifauna, estructura de las comunidades, cambio estacional, migración.

Jiménez-Pérez, L. C., H. de la Cueva, R. F. Molina-Peralta \& A. Estrada-Ramírez. 2009. Birds of Estero de Punta Banda, Baja California, Mexico. Acta Zoológica Mexicana (n. s.), 25(3): 589-608. ABSTRACT. Twenty four bird identification and counting campaigns were carried out at Estero de Punta Banda, Baja California, Mexico between March 2004 and March 2005, in six representative environmental units. Thirty-five bird families were registered, including 104 species. Sixty-four species were associated with aquatic environments. Both, aquatic and terrestrial communities showed clear temporal patterns with three groups associated with seasonal changes. Twelve of these species

Recibido: 13/02/2009; aceptado: 22/09/2009. 
are listed as protected in the Mexican environmental legislation. We found evidences of the reproductive success of the Clapper Rail and the Black-necked Stilt populations in disturbed areas. These results indicated that the Estero de Punta Banda remains an important reproduction-feeding area for many resident and migratory birds. However, the rapid urban development of the City of Ensenada, water pollution, and illegal use of its natural resources, threaten the survival of Estero Punta Banda and its bird species.

Keywords: Bird biodiversity, community structure, seasonal changes, migration.

\section{INTRODUCCIÓN}

El Estero de Punta Banda (EPB) es un cuerpo de agua elongado de aproximadamente $7 \mathrm{~km}$ de largo. Recientemente fue designado Sitio de Importancia Internacional para las Aves Migratorias por la Convención Ramsar (Valencia 2005). Esa designación se deriva de la gran variedad de hábitats y riqueza biológica que contiene. Entre los hábitats sobresalen sus amplias planicies de marea y planicies fangosas. La marismas ocupan un área aproximada de 326 ha, mientras que las planicies fangosas se extienden 170 ha (http://proesteros.cicese.mx). Además, contiene 78 ha de dunas de arenas finas, así como pequeñas secciones del litoral de playas arenosas, canales y charcas de marea.

En las últimas décadas se han construido desarrollos turísticos y residenciales (Figura 1), así como un dique que aisló parte del cuerpo de agua, formando una pequeña laguna con una superficie de aproximadamente 10 ha (en lo sucesivo, este cuerpo de agua será denominado la "Lagunita"). La proliferación de asentamientos humanos en los alrededores ha ocasionado importantes pérdidas de hábitats, principalmente en la barra arenosa que separa al estero de mar abierto. El crecimiento de los asentamientos humanos en la rivera sur del estero es otro elemento de disturbio que ha venido afectando gradualmente las condiciones naturales del estero. Ambas situaciones han ocasionado un aumento en el número de personas y mascotas que acuden de manera frecuente a las diferentes áreas del estero, lo que pudiera estar amenazando la persistencia de algunas poblaciones naturales.

Escofet et al. (1988) mostraron que EPB es un área de protección, alimentación y reproducción de muchas especies de aves, entre ellas 4 que se encontraban enlistadas en la categoría de especies en peligro de extinción. Asimismo, se hizo hincapié en la importancia de EPB como enlace en la ruta de migración del Pacifico (Palacios et al. 1991). Sin embargo, no se ha determinado si los cambios locales asociados con el desarrollo de los asentamientos humanos cercanos y los cambios climáticos de escala global han afectado a las aves del EPB. En este estudio se identifican las especies de aves más representativas del EPB, así como sus patrones de variación espaciotemporal a lo largo de un ciclo anual. Asimismo, se hace una relación de las especies enlistadas en alguna categoría de protección, así como los hábitats donde se observaron. Además, se hacen sugerencias que pudieran ser implementadas para asegurar la conservación de sus poblaciones. 


\section{MATERIAL Y MÉTODOS}

Entre enero y febrero de 2004 se efectuaron salidas de reconocimiento de las diferentes unidades ambientales que conforman el EPB así como para familiarizarnos con las aves presentes en esas áreas. Sobre la base de las observaciones efectuadas, se seleccionaron 6 áreas que se consideraron representativas del EPB (Figura 1): la boca del estero (B), dos caminos vecinales $(\mathrm{Cv})$, la marisma principal (Map), la Lagunita (L), la barra arenosa (Ba) y el canal principal (Cp).

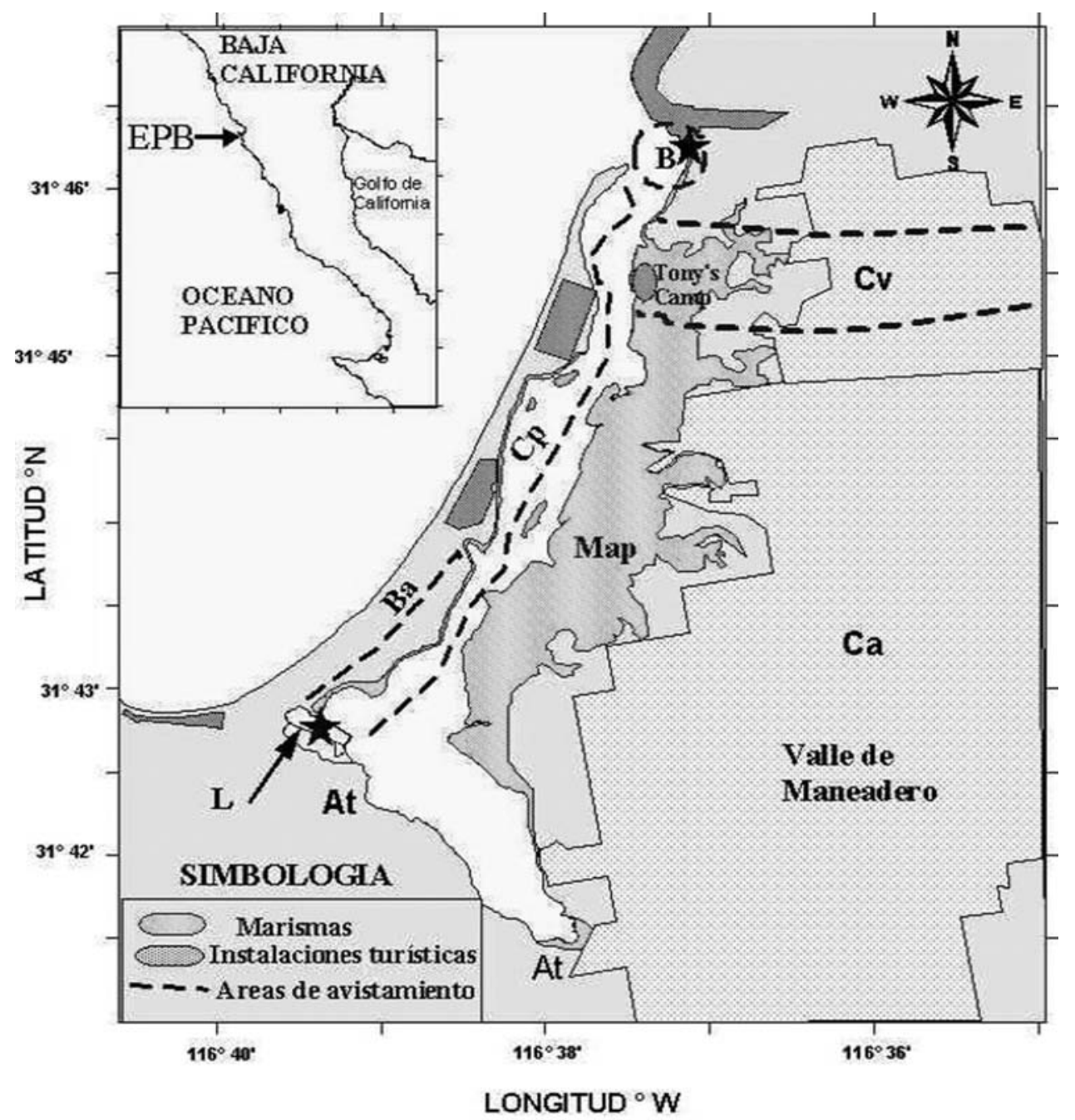

Figura 1. Localización del Estero de Punta Banda. Las dos estrellas indican la localización nidos del Rallus longirostris e Himantopus mexicanus. 
Posteriormente se elaboró un registro sistemático de la avifauna, siguiendo una misma secuencia: mensualmente se efectuaron recorridos a pie para la zona $\mathrm{B}$ en condiciones de marea baja, siguiendo el contorno de un corredor turístico situado en el borde del humedal y censando las aves de las unidades de marisma, charcas y planicies de marea, así como de un dique de cantos rodados (Dcr) que se localizan en esa zona. Después de haber inspeccionado la zona $\mathrm{B}$ se procedió a recorrer los $\mathrm{Cv}$ a bordo de un vehículo terrestre. Estos monitoreos se efectuaron a lo largo de dos transectos ubicados entre la carretera transpeninsular y el inicio de la Map. Para caracterizar la avifauna de la zona $\mathrm{Cv}$ se efectuaron avistamientos desde ambos lados del vehiculo así como recorridos a pie a lo largo dos parcelas de marisma de aproximadamente 1 ha. Una de esas parcelas de marisma esta situada en el límite norte de la Map y la otra en el límite sur del campo turístico Tony's Camp. En cada una de estas zonas se registró la avifauna de los canales de marea.

Los registros mensuales de la avifauna de las zonas $\mathrm{Cp}$, Ba y L se efectuaron dos semanas después, cuando las condiciones de marea alta permitieron navegar a lo largo del canal principal. Esta secuencia de monitoreo dio inicio recorriendo el $\mathrm{Cp}$ a bordo de una embarcación menor de $6 \mathrm{~m}$ de eslora con un motor fuera de borda, hasta las inmediaciones de la Lagunita; a continuación se efectuó la inspección del tramo de la $\mathrm{Ba}$, llevando a cabo recorridos a bordo de un vehículo terrestre a lo largo de la carretera de asfalto, y caminando entre la vegetación cuando se detectó la presencia de alguna ave. El monitoreo de L se efectuó haciendo observaciones desde el dique y caminando en el borde de la delgada franja de marismas y zona de transición allí existentes; además, se efectuaron observaciones adicionales del avifauna en un salitral adyacente a la L así como de los campos agrícolas (Ca), así como de las áreas terrestres adyacentes (At).

La cuantificación de la avifauna se realizó aplicando dos métodos de estudio; en las zonas B, Mp y L se aplicó el método de conteos puntuales (Bibby et al. 1993). En las dos primeras zonas se seleccionaron parcelas de aproximadamente 1 ha, mientras que en la L se inspeccionó todo el cuerpo de agua de aproximadamente 4 ha y sus orillas. Las evaluaciones de la avifauna de $\mathrm{Cv}, \mathrm{Ba}$ y $\mathrm{Cp}$ se efectuaron aplicando el método de transectos (Bibby et al. 1993). Los Cv tuvieron una extensión aproximada de 4 a $5 \mathrm{~km}$ y cubrieron una amplitud de aproximadamente $20 \mathrm{~m}$ hacia cada lado de los caminos. El transecto de la barra inspeccionado se extiende desde el embarcadero de Baja Beach y Tennis Club y la L. La cuantificación de las especies se realizó contando individuos, sin hacer aproximaciones de grupos. La identificación de las aves se realizó utilizando binoculares 7 x 35 y guías de campo (Cogswell 1977, Peterson y Chalif 1989, ESSA 2000). La nomenclatura y arreglo sistemático de las especies está basado en la propuesta de la American Ornithologist's Union (1998). Posteriormente se crearon bases de datos y se procesaron utilizando técnicas de análisis multivariadas contenidas en el programa PRIMER (Clarke y Warwick 2001). 
Las rutinas usadas fueron análisis de similitud porcentual (SIMPER) global, análisis de agrupamientos y análisis de similitudes (ANOSIM). La aplicación del SIMPER permitió identificar las especies más representativas. Estas especies fueron seleccionadas, considerando como tales a aquellas que contribuyeron con el $90 \%$ de la similitud observada en todo el estudio. Debido a la falta de normalidad de la distribución de los datos, se utilizó la transformación raíz cuadrada. A continuación, se construyeron dendrogramas basados en los índices de Bray-Curtis, aplicando el método de conglomerados jerárquicos con la opción de enlace promedio de grupo (Clarke y Warwick 2001).

El dendrograma fue analizado para identificar las diferentes agrupaciones. Para determinar si las diferencias observadas entre esos grupos eran significativas, se aplicó el análisis de similitudes (ANOSIM) como análisis discriminante. Cuando las diferencias fueron significativas, este análisis fue seguido por un análisis del porcentaje de similitud (SIMPER), procedimiento que determina la contribución de cada especie, calculando la disimilaridad promedio de Bray-Curtis $\left(\% o=100-\mathrm{d}_{\text {Bray- }}\right.$ Curtis) entre todos los pares de muestras.

Para identificar los cambios temporales en la estructura comunitaria se efectuó una evaluación cuantitativa de la diversidad y riqueza de especies. En primer término, se elaboró un inventario general de las especies registradas, pretendiendo caracterizarlas como acuáticas y terrestres, dependiendo de su afinidad por los hábitats. Posteriormente se determinó la riqueza de especies (S) y se estimaron los índices de diversidad de especies por censo mensual. La diversidad de especies fue estimada con el índice de Shannon, H (Zar 1984). Para identificar posibles cambios temporales de más largo plazo, los datos de presencia y ausencia generados en este estudio fueron comparados con los de Escofet et al. (1988) aplicando un ANOSIM.

\section{RESULTADOS.}

Se identificaron 10,948 individuos pertenecientes a 35 familias que incluyeron a 104 especies de las cuales 64 están asociadas con el ambiente acuático (Apéndice 1). Entre ellas, se encuentran quince especies de anseriformes, una de falconiformes, 2 gallinitas de agua, 3 pelicaniformes, 2 podiciformes, una gaviforme, 7 ciconiformes y 32 charadriformes. Entre las 40 especies de afinidad terrestre se registraron 8 Falconiformes (un águila, 2 aguilillas, 4 halcones, un milano), dos palomas (Zenaida macroura y Columba livia), un galliforme (Callipepla californica, codorniz de California), un ibis (Plegadis chihi) y 28 passeriformes (Apéndice 1).

Doce especies registradas en este estudio se encuentran incluidas en alguna categoría de protección por la NOM-059. A partir de la frecuencia de observación registrada en el apéndice 1 se puede deducir que dos de esas especies son residentes del estero y nueve son migratorias. Las especies residentes son el gorrión sabanero Passerculus sandwichensis, una subespecie amenazada (condición A en la NOM- 
059) y el rascón picudo Rallus longirostris. P. sandwichensis presentó una distribución amplia, ocupando la zona de la marisma alta donde predominan las matas de Salicornia sp. o del pasto playero (Monanthochloe littoralis). Las principales poblaciones del rascón picudo se encontraron en la parte baja de la Map (entre plantas de Spartina sp.), pero también se les observó en la unidad principal de marisma de la B, así como cruzar los canales y planicies de marea de esa zona.

Entre las nueve aves migratorias protegidas detectadas en este estudio se encuentran cinco poblaciones acuáticas y cuatro terrestres. Entre las especies acuáticas el charrán mínimo Sterna antillarun, el charrán elegante Sterna elegans, la gaviota ploma Larus heermanni y el ganso de collar Branta bernicla y la garceta rojiza Egretta rufescens. S. antillarun se detectó forrajeando en la zona de la boca del estero, así como volando a lo largo del canal principal entre mayo y agosto. Por su parte, $L$. heermanni se registró parada o volando exclusivamente en la boca entre agosto y diciembre, mientras $B$. bernicla se observó entre octubre y marzo, nadando en las inmediaciones de la desembocadura del Arroyo San Carlos, así como volando o caminando en la orilla de la Map. E. rufescens, especie protegida (condición Pr) se detectó entre abril y septiembre, en las charcas y canales de marea en Map y B.

Las aves terrestres que aparecen en la NOM-059 ECOL 2001 son el búho cuerno corto Asio flammeus, el cual se registró en febrero y marzo de 2005; el águila real Aquila chrysaetos, que se le vio parada en la cresta de una duna, cerca de la punta de la B en enero de 2005; el halcón peregrino Falco peregrinus; el halcón mexicano $F$. mexicanus, individuos juveniles de la aguililla cola roja Buteo jamaiciensis e individuos juveniles de la aguililla de Swainson Buteo swainsoni. Estos últimos fueron registrados ocasionalmente en los caminos que conducen hacia la marisma del campo de aeromodelismo y Tony's Camp.

En términos de abundancia relativa, las aves acuáticas representaron más del 90\% de las aves observadas en cada salida de campo (Figura 2). Los resultados del SIMPER demostraron que Catoptrophorus semipalmatus, Limosa fedoa, Larus occidentalis, Rynchops. niger, Himantopus mexicanus, Pelecanus occidentalis, Phalacrocorax autitus, Fulica americana y Egretta. thula fueron las especies que mejor caracterizan la avifauna del EPB. C. semipalmatus y L. fedoa alcanzaron niveles de abundancia superiores a los 250 individuos en mayo. Les siguieron en el orden jerárquico de abundancia $P$. occidentalis, $P$. auritus, $L$. occidentalis y $R$. niger. L. occidentalis y $R$. niger alcanzaron sus mayores abundancias en marzo y abril (200 y 175 individuos, respectivamente). Por su parte, P. occidentalis presentó su mayor abundancia en julio y agosto (150 individuos), mientras que $P$. auritus alcanzó ese nivel de abundancia en el periodo de octubre a diciembre. En un tercer orden de abundancia se localizaron las F. americana, Anas americana y $H$. mexicanus, con niveles máximos de abundancia ligeramente menores a 100 individuos. 


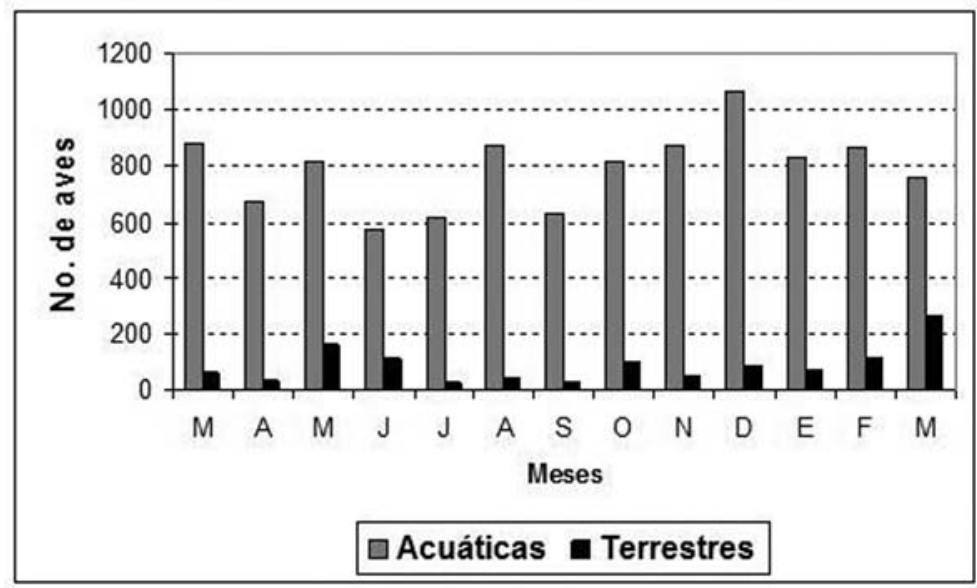

Figura 2. Número de aves registradas en el Estero de Punta Banda, Baja California entre marzo de 2004 y marzo de 2005.

La riqueza y diversidad de las especies acuáticas presentó un valor relativamente alto en marzo de 2004, pero presentó una tendencia a disminuir durante los meses de verano. Posteriormente aumentó gradualmente hasta alcanzar los valores altos en febrero y marzo de 2005. Por el contrario, la riqueza y diversidad de especies terrestres presentó una mayor variación a lo largo del periodo estudiado (Figura 3a y 3b). Ambos parámetros fueron más altos en las especies acuáticas.

El análisis de agrupamientos de las especies acuáticas indicó la existencia de 3 grupos (Figura 4). Un grupo pequeño constituido por los censos efectuados en marzo y abril de 2004 (grupo transicional), otro por los censos efectuados entre mayo y septiembre de 2004 (grupo estival), mientras que el tercer grupo (grupo invernal) estuvo constituido por la avifauna registrada entre octubre y febrero. Las especies más representativas del grupo estival fueron L. fedoa y C. semipalmatus (Figura 5a), pero también se detectaron importantes poblaciones de $P$. occidentalis, $L$. occidentalis y $R$. niger. $L$. fedoa y $C$. semipalmatus continuaron estando entre las especies más representativas del grupo invernal. Sin embargo, fueron desplazados del primer lugar del orden jerárquico de abundancia por Melanitta perspicillata. En este grupo, F. americana y A. americana ocuparon los puestos cuarto y quinto, desplazando a $P$. occidentalis, $P$. auritus, $L$. occidentalis y $R$. niger hasta los sitios de menor importancia (Figura $5 b$ ). Las especies más representativas del grupo de transición fueron $C$. semipalmatus, L. occidentalis, $R$. niger, F. americana y L. fedoa (Figura 5c). La estructura comunitaria de este grupo fue dominada por las especies residentes. Sin embargo, también se registró una proporción importante de especies migratorias e invernantes (Figura 6). 


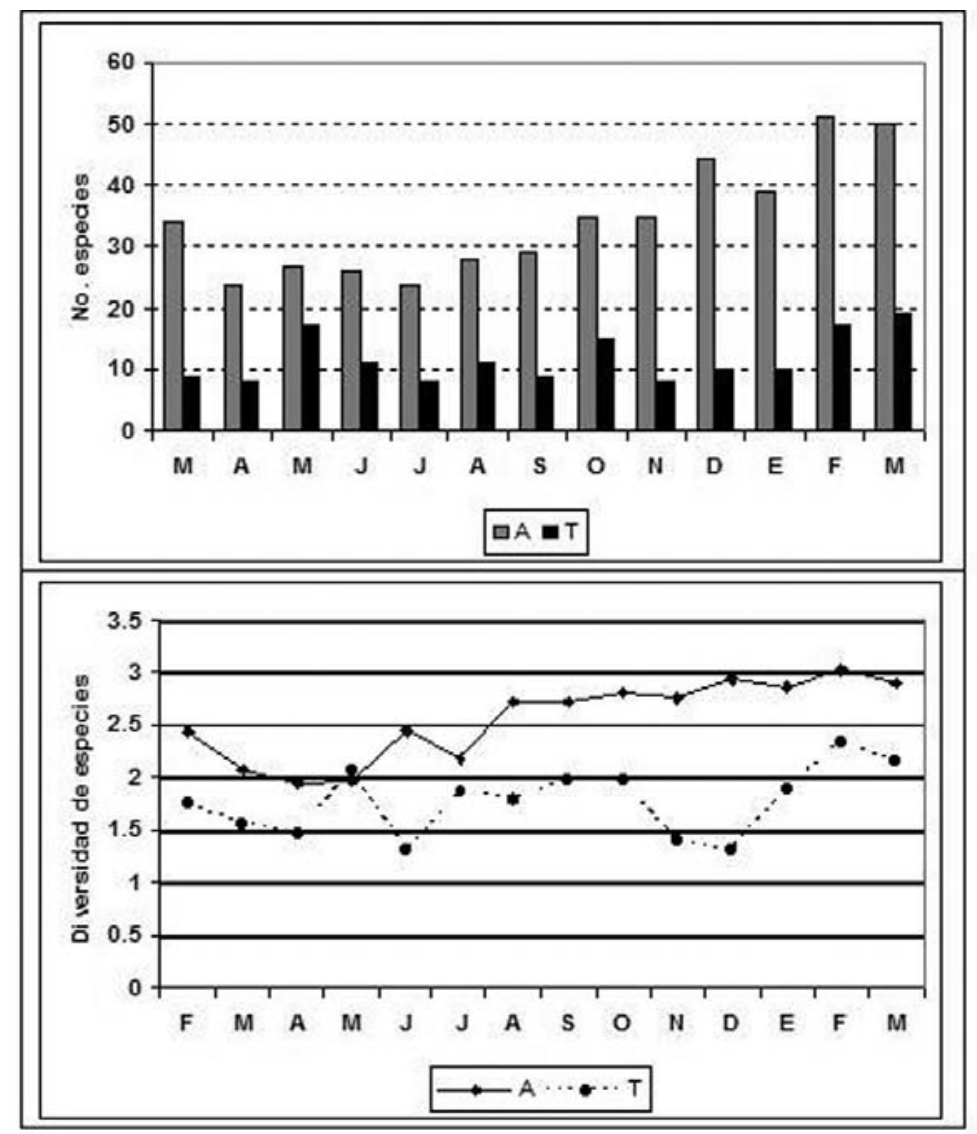

Figura 3. Riqueza y diversidad de especies de la avifauna del Estero de Punta Banda, Baja California registradas entre marzo de 2004 y marzo de 2005. A = especies acuáticas; $\mathbf{T}=$ especies terrestres.

En este estudio se registraron evidencias del éxito reproductivo de especies residentes del estero. El 19 de mayo se detectó a $R$. longirostris anidando en una marisma situada en la B. Tres días después se regresó al sitio, observándose que la pareja de aves tenía 2 polluelos. Durante mayo se registraron numerosos nidos de $H$. mexicanus con huevos en diferentes sitios tanto en el margen norte de L como en la orilla sur del salitral; en las siguientes semanas se observaron a sus polluelos caminando cerca del agua.

Las principales poblaciones acuáticas exhibieron una importante variabilidad en sus patrones de distribución en respuesta a las condiciones físicas cambiantes del estero. Por ejemplo, las diferentes especies de chorlos y Numenius americanus deambulaban sobre las planicies fango-arenosas durante la bajamar y se replegaban 


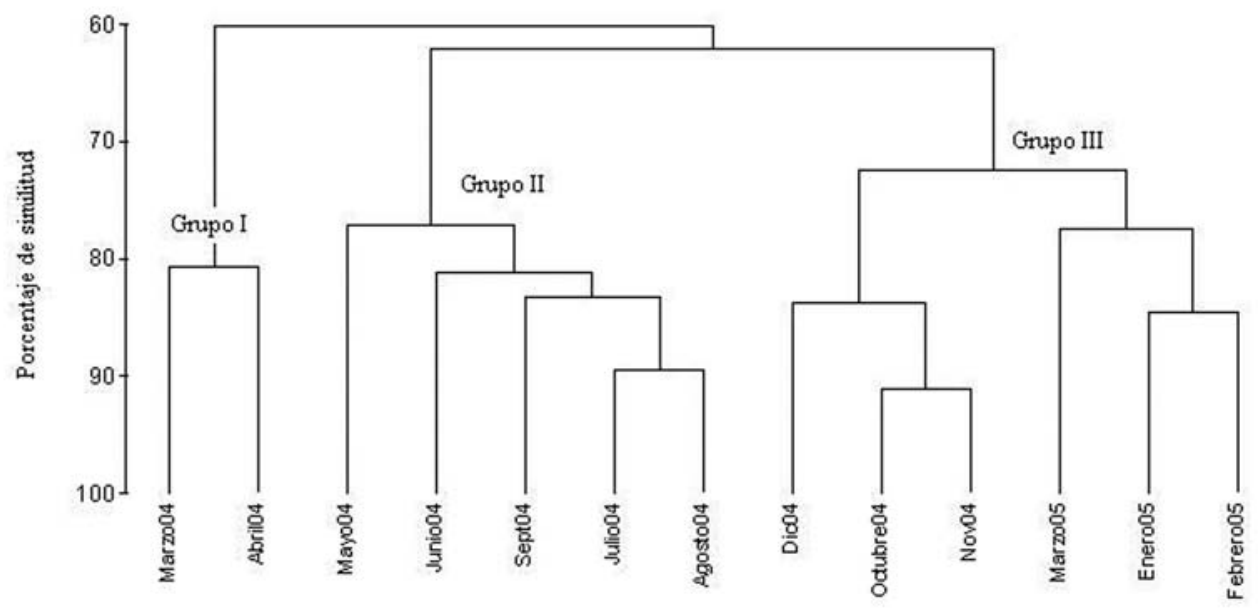

Figura 4. Análisis de conglomerados de la avifauna acuática del Estero de Punta Banda, Baja California clasificada por mes de avistamiento.

hacia las marismas al subir el nivel del agua. Durante la bajamar, C. semipalmatus y L. fedoa presentaban una tendencia a conglomerarse en la barra (B), formando grupos mixtos de cientos de aves. Permanecían alimentándose a lo largo de la orilla, pero al subir la marea, se replegaban hacia las partes altas. Cuando el nivel del agua aumentaba aún más, se dispersaban a lo largo del estero, situándose en pequeños grupos sobre el borde de las marismas. Este comportamiento se registró en todas las salidas de campo efectuadas en marea baja.

$R$. niger mostró un comportamiento similar al observado en $C$. semipalmatus y $L$. fedoa; en bajamar tendían a establecerse en las planicies fango-arenosas cercanas a la $\mathrm{B}$, pero cambiaban su posición de acuerdo con el ritmo de la marea. A veces se paraban entre los C. semipalmatus y L. fedoa, pero también se les observó formando un grupo aislado. Cuando eran perturbados volaban hacia la punta de la barra arenosa y se paraban junto al grupo de $L$. occidentalis, $P$. occidentalis y $P$. auritus que usualmente dominaban el avifauna de ese sitio. $P$. occidentalis también presentó un comportamiento asociado con la dinámica de las aguas. En marea alta, se paraban en la punta de la barra arenosa, pero al bajar el nivel de las aguas volaba hacia un banco fango-arenoso que se forma cerca del canal principal.

Por su parte, F. americana, 6 especies de anátidos, 2 de faralopos (Phalaropus fulicaria y P. lobatus), Recurvirostra americana y $H$. mexicanus, optaron por las aguas más tranquilas de L o del Salitral. F. americana, los anátidos, $P$. fulicaria y $P$. lobatus se registraron exclusivamente en $\mathrm{L}$, mientras que $H$. mexicanus y $R$. americana ocuparon tanto las orillas de ese cuerpo de agua, como la charca del 
Salitral adyacente. En contraste, $M$. perspicillata se registró exclusivamente a lo largo del canal principal, mientras que Mergus serrator y M. merganser, así como Aechmophorus accidentalis y Podiceps nigricollis (somormujos) se registraron tanto en el canal principal como en la Lagunita (Apéndice 1).
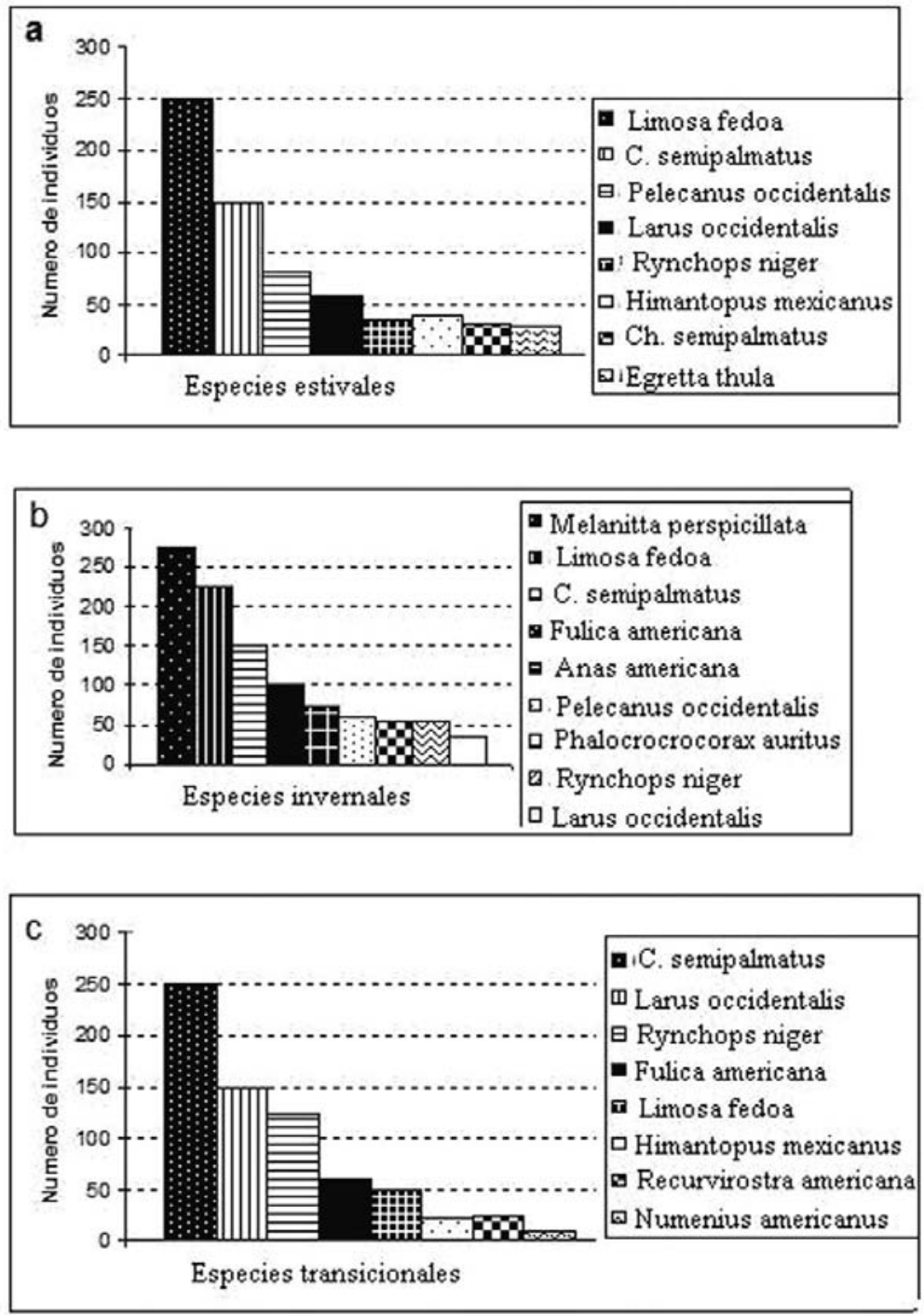

Figura 5. Principales poblaciones de la avifauna de los grupos estival (a), invernal (b) y de transición (c). 


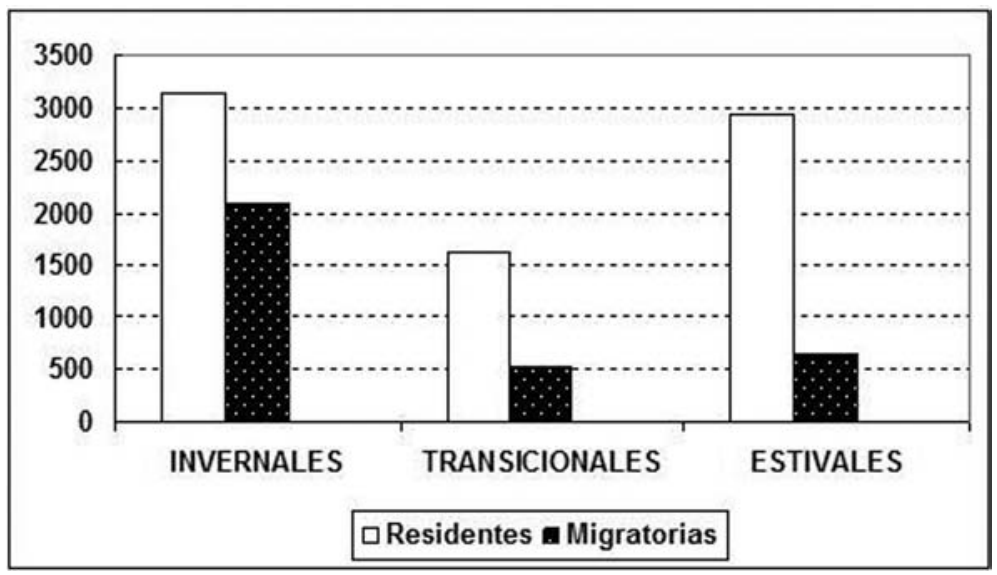

Figura 6. Número total de individuos observados en el Estero de Punta Banda entre marzo de 2004 y marzo de 2005.

Por su parte, los caminos de acceso a las marismas, constituyen zonas de alimentación de una gran variedad de aves terrestres que dependen de la vegetación o de insectos que sobre ellas viven: Z. macroura, C. livia, Sturnus vulgaris y Euphagus cyanocephalus, pero igualmente sirven como cotos de caza de algunas aves rapaces: F. mexicanus, F. peregrinus, B. jamaiciensis y B. swainsoni. La barra arenosa es utilizada como zona de reproducción de $S$. antillarun y constituye un hábitat importante para varias especies; entre ellas C. californica, Sturnella neglecta y Mymus polyglottos. Además, constituye un coto de caza de Falco sparverius y Asio flammeus.

Las especies terrestres más representativas fueron C. livia, Z. macroura, S. vulgaris, E. cyanocephalus y Petrochelidon pyrrhonota. En conjunto, estas 5 especies contribuyeron con el $73 \%$ de la similitud estimada entre las especies terrestres. Otras especies menos abundantes, pero que se detectaron todos los meses y con bastante frecuencia fueron $M$. polyglottos y Carpodacus mexicanus. $C$. californica, S. neglecta, Cathartes aura y Corvus corax se registraron en más del $50 \%$ de los censos, pero en bajas cantidades.

La estructura comunitaria de las aves terrestres presentó una secuencia temporal similar a la registrada en las especies acuáticas (Figura 7). El grupo de transición estuvo conformado por los censos de marzo y abril de 2004, el grupo invernal por los censos efectuados entre noviembre de 2004 y febrero de 2005. Por su parte, el grupo estival estuvo integrado por los censos efectuados entre mayo y septiembre de 2004. Los censos de octubre no cayeron en ninguno de esos agrupamientos, pero estuvieron mas cercanamente relacionados con el grupo invernal. 


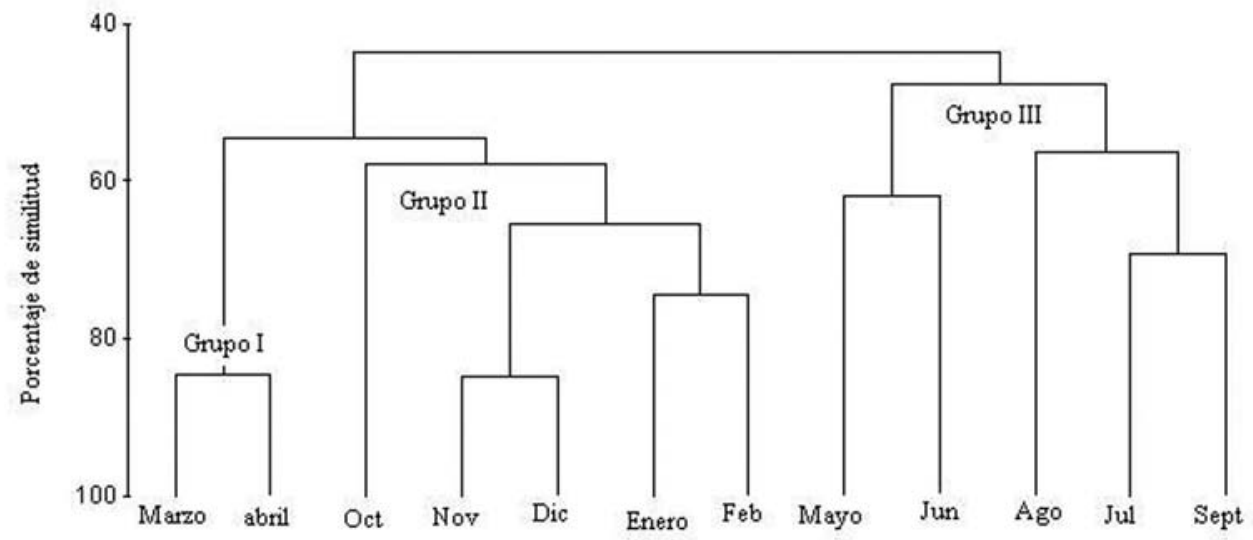

Figura 7. Análisis de conglomerados de la avifauna terrestre del Estero de Punta Banda, Baja California observada entre marzo de 2004 y marzo de 2005.

La disimilitud porcentual promedio detectada entre los grupos invernal y estival de las especies terrestres fue de $74 \%$, siendo aproximadamente el $80 \%$ de ella explicada por las variaciones en los patrones de abundancia de 9 especies; 4 de ellas: $C$. livia, $S$. vulgaris, Agelaius phoeniceus y $F$. sparverius fueron mas abundantes en el grupo invernal, mientras que las 5 restantes: $P$. pyrronota, Hirundo rustica y Petrochelidon fulva, Z. macroura y $C$. aura fueron mas abundantes en el grupo estival. El grupo de transición estuvo constituido principalmente por $C$. livia, $P$. pyrrhonota, $C$. californica y Molotus ater. C. livia y $M$. ater se observaron sobre los campos agrícolas situados en las inmediaciones de la marisma principal. Además, durante el invierno sobresalió la presencia de poblaciones de A. flammeus y Atene cunicularia. Los primeros fueron detectados en la barra del estero, mientras que los segundos se detectaron en la barra arenosa que corta la marisma de L.

\section{DISCUSIÓN}

Los resultados obtenidos en este estudio indican que el EPB continua siendo un ecosistema con una gran importancia para la avifauna regional, a pesar de los cambios ambientales registrados en las últimas décadas. El cambio estacional detectado en la estructura de las comunidades acuáticas y terrestres pone de manifiesto la importancia de las especies migratorias en la riqueza biológica del EPB. La presencia, entre octubre y marzo, de 2 anseriformes y 13 anátidos, contribuye a realzar la importancia del EPB como hábitat de invierno para esas especies. Este ecosistema forma parte del corredor del Pacífico que es utilizado por millones de aves para migrar entre sus áreas de reproducción en el Ártico y subártico 
y las áreas de hibernación de latitudes medias (Escofet et al. 1988). En consecuencia, constituye uno de los paradores que utilizan estas aves migratorias antes de continuar su viaje y proporciona un importante apoyo a las poblaciones que dependen de los hábitats intermareales o praderas de pastos marinos para obtener alimento (Zedler et al. 1992).

La presencia de 12 especies listadas en la NOM-059-ECOL-2001 representa un punto importante, y debe ser considerada en la adopción de lineamientos en materia de equilibrio ecológico y protección al medio marino, implementación de medidas y programas de preservación y restauración del equilibrio del ecosistema así como en las políticas de desarrollo urbano futuras. La presencia de estas especies, así como la detección de problemas ambientales, indica la necesidad urgente de revisar los planes de desarrollo municipales e implementar mecanismos de protección que permitan asegurar la supervivencia de esas poblaciones, pues la urbanización de grandes áreas en las inmediaciones de la marisma principal constituye una amenaza para la supervivencia de hábitats y especies.

Las especies más vulnerables a la implementación de políticas de desarrollo urbano sin medidas de protección son las especies residentes, porque los núcleos de sus poblaciones se encuentran cerca de áreas donde se están asentando nuevos fraccionamientos. Esta situación de riesgo adquiere una mayor dimensión si se considera que frecuentemente se detectaron perros caminando libremente por las marismas o escarbando en las madrigueras de la fauna silvestre, a pesar del relativo aislamiento que aun persiste del núcleo urbano. De continuar esta tendencia, el desarrollo urbano podría constituir un serio problema para la seguridad de los nidos, huevos y polluelos de las especies mencionadas. Del mismo modo, la lotificación y venta de terrenos en una amplia zona de transición de la barra arenosa que es utilizada como hábitat por las poblaciones migratorias de A. flammeus, una especie protegida podría constituir una seria amenaza para esas poblaciones.

Por otra parte, la presencia de nidos con huevos y polluelos de $R$. longirostris en la marisma de la boca del estero permitieron documentar el evento, así como aportar evidencias de que no siempre las estructuras hechas por el hombre afectan negativamente a las poblaciones animales, pues el nido estaba a $20 \mathrm{~m}$ de un andador construido para los huéspedes del complejo turístico. Aparentemente, el aislamiento relativo del área, asociado con el fuerte mecanismo de vigilancia y control de la zona implementado por la administración del Hotel Estero Beach está favoreciendo la supervivencia de esta especie. Una situación similar se presentó tanto en L, como en El Salitral, donde la presencia de nidos con huevos y polluelos de $H$. mexicanus indicaron que esos hábitats tienen una importante función ecológica en la supervivencia local de estas poblaciones.

En general, los resultados obtenidos en este estudio indican que a pesar de las múltiples modificaciones de que ha sido objeto en las últimas décadas, el EPB 
continúa siendo un ecosistema importante como zona de descanso, alimentación y reproducción de muchas especies de aves residentes y migratorias. Por su parte, la existencia de patrones espaciales revela no solamente la importancia de algunos hábitats como zonas de alimentación, descanso y anidación, sino que reflejan la naturaleza dinámica del EPB y su enorme importancia para la vida silvestre. Sin embargo, el acelerado desarrollo urbano de la ciudad de Ensenada amenaza con dañar posiblemente de manera irreversible la integridad de algunos hábitats. Este riesgo parece ser particularmente importante para la marisma principal, un hábitat que se encuentra ubicado en las inmediaciones de un asentamiento humano reciente. Así como para la zona de transición ubicada en la barra principal, pues una parte importante ya ha sido lotificada y puesta a la venta.

Por otra parte, resultó inesperada la tendencia que manifestaron las poblaciones de la $F$. americana, $R$. americana y $H$. mexicanus, así como las 5 especies de patos y $P$. fulicaria y $P$. lobatus por ocupar principalmente las aguas de la L, pues esta zona está siendo sometida de manera constante a la disposición clandestina de aguas residuales. Sin embargo, la presencia constante de $P$. occidentalis, $P$. auritus y Sterna spp. en una playa fangosa que se localiza en la parte central de ese sistema sugiere que en esa zona existe una buena disponibilidad de alimento que pudiera estar relacionada con el efecto fertilizador de las aguas residuales. Aparentemente, la disposición de esas aguas contribuye a la fertilización de L. En consecuencia, parece ser que el éxito reproductivo de la población de H. mexicanus, también representa un efecto de la mayor disponibilidad de alimento asociada con la disposición de aguas residuales. Esta relación entre disponibilidad de alimento y éxito reproductivo de algunas aves ha sido bien documentada en muchos estudios realizados en poblaciones de diferentes partes del mundo (p. ej. Anderson et al. 1982; Schaffner 1986; Springer et al. 1986; Cairns 1987). Sin embargo, también es posible que esta situación se deba al menos en parte, a que el mal olor que prevalece en la zona favorece el aislamiento del lugar. Pareciera que lo que para nosotros representa un problema (la contaminación de las aguas L) para las aves significa una oportunidad de refugio y alimento.

La comparación de la estructura comunitaria detectada en este estudio con la reportada por Escofet et al. (1988) indicó la existencia de cambios significativos. Estas diferencias sugieren la existencia de cambios temporales de mayor escala que no han sido documentados. Es posible que algunos de estos cambios estén relacionados con variables físicas como el cambio climático global u otras variables físicas y ecológicas que no es posible detectar sin un monitoreo constante de ellas. Sin embargo, también es posible que pudieran estar asociados con las estrategias de muestreo. Por ejemplo, el registro de 104 especies en este estudio, puede ser considerado alto, si se compara con las 69 y 23 especies reportadas respectivamente por Nishikawa (1983) y Escofet et al. (1988). Sin embargo, esto no significa 
necesariamente que en las últimas décadas haya aumentado la riqueza faunística. Creemos que estos resultados resultaron de la mayor continuidad y la estrategia de avistamiento implementada en este estudio. El monitoreo de L demostró ser un factor clave, ya que en este sitio se efectuaron 9 de los 23 registros inéditos; entre ellas, 4 especies de patos y una cerceta de la familia Anatinae: Anas acuta, A. americana, A. clypeatra, A. cyanoptera y A. discor. Además, sólo en esta zona se registraron a $F$. americana, Larus delawarensis, P. lobatus y P. fulicaria.

La incorporación del canal principal como zona de avistamiento permitió registrar 4 especies que no fueron reportadas por Escofet et al. (1988): B. bernicla, M. serrator, M. merganser y Gavia arctica. De igual manera, los nuevos registros de especies terrestres parecen estar relacionada con la selección de los 2 transectos de zonas de transición. El monitoreo de los caminos de acceso a las marismas permitió incorporar al elenco 3 especies de aves rapaces: $F$. peregrinus, e individuos jóvenes de $F$. mexicanus y $B$. swainsoni.

Por el contrario, no se detectó la presencia de 10 de las especies reportadas por Escofet et al. (1988), Melanitta nigra, Butorides virescens, Actitis macularius y Heteroscelus incanum. Se estima que la ausencia de estas especies en nuestro estudio, pudiese estar relacionada con sus bajos niveles de abundancia en la zona. Una situación similar pudiera explicar la ausencia de las gaviotas Larus californicus y Larus argentatus. Sin embargo, la ausencia de Eremophila alpestris y Campylorhynchus brunneicapilus esta asociada a la menor intensidad de monitoreo de los hábitats terrestres. Fulmarus glacialis, es otra de las especies reportadas por Escofet et al. (1988). que no se detectó en este estudio. Sin embargo, su presencia en el área ha sido confirmada en otras instancias. Por ejemplo, por la ocurrencia de una mortandad de cientos de especimenes varados en la barra del estero entre el 5 y 9 de noviembre de 2003 (Jiménez Pérez 2003).

\section{RECOMENDACIONES DE CONSERVACIÓN Y MANEJO}

En el Programa de Desarrollo Urbano del Centro de Población de Ensenada, se designó a las marismas, planicies fangosas y la playa situada en la punta de la barra arenosa de nuestra área de estudio como áreas de "Protección con uso pasivo", mientras que el matorral costero de la punta de la barra, los salitrales y el canal principal fueron designados como zonas de "Protección con uso activo" (Periódico Oficial del Estado de Baja California 1995). Sin embargo, estas declaraciones no se han logrado concretar pues, como en muchos otros sitos de interés de conservación, no se implementaron programas de vigilancia y control de las actividades. Una de las pocas evidencias que persisten de esas acciones de conservación es la presencia de letreros en una zona de matorral costero de aproximadamente 20 ha, situada cerca de la punta de la barra. Esta reserva ecológica cuenta con senderos interpretativos con carteles explicativos de flora y fauna del lugar. Sin embargo, la falta de vigilancia y control ha ocasionado que la 
mayor parte de la zona de dunas se encuentre invadida por especies exóticas y no haya control sobre los animales domésticos que ingresan a la zona.

Las necesidades más urgentes de protección en el EPB es la erradicación de fauna nociva invasora de la zona de marismas. Durante nuestras observaciones se detectó la presencia de gatos en las inmediaciones de los nidos de $R$. longirostris, así como perros escarbando entre las madrigueras de la marisma alta o vagando en las inmediaciones de los nidos de $H$. mexicanus. Sería muy conveniente la presencia de la autoridad ambiental, o en su caso municipal, obligara a los propietarios a mantener sus mascotas dentro de su propiedad. Es decir, que se les obligue a cercar sus terrenos de manera apropiada. En la actualidad el nivel de depredación por parte de estos animales parece ser bajo, ya que durante nuestros recorridos encontramos pocos pollos de $R$. longirostris muertos, y no fue posible determinar si la causa principal era la depredación por la fauna doméstica. Sin embargo, el problema podría aumentar en el futuro, pues tanto en las cercanías de la Map, como en la parte interna de la B se están ofreciendo terrenos a la venta. Otra medida de conservación importante sería un cerco perimetral que separe la marisma de las áreas que están siendo ocupadas por nuevos colonos. Idealmente se maximizaría la conservación si se formara una figura jurídica o un fideicomiso que asumiera el control de las actividades que allí se realizan. Este organismo podría regular las actividades de investigación y monitoreo de los diferentes elementos bióticos y abióticos del ecosistema.

Nuestros resultados permiten concluir que las alteraciones del hábitat en la Map, las planicies lodosas y el Canal principal no han sido significativas en cuanto a la riqueza de aves que se registra. Sin embargo, en B, donde la mayor parte de su extensión ha sido ocupada por conjuntos habitacionales hay menor disponibilidad de hábitat y consecuentemente menos aves. Por otra parte, la presencia de fauna introducida nociva en la marisma constituye un fuerte riesgo para la conservación de las poblaciones de aves que allí habitan o que la hacen su refugio invernal. Consideramos que la agenda de investigación debe incluir la evaluación del tamaño y dinámica poblacional de las especies protegidas, así como la evaluación de la presión por depredación de la fauna nociva. Por otra parte, también es importante efectuar una evaluación de las poblaciones de otros grupos zoológicos como insectos y otros artrópodos, mamíferos pequeños y reptiles.

AGRADECIMIENTOS. Agradecemos a Jorge Vega, Fernando González y Héctor Gómez de Silva por sus valiosos comentarios a la versión original. Asimismo, agradecemos las facilidades brindadas en el uso de sus embarcaderos por los Sres. Luis Alberto Novelo y Jorge Cortina, administradores del Hotel Estero Beach y del Baja Beach and Tennis Club, respectivamente. El presente trabajo formó parte del proyecto "Caracterización de áreas sensibles a la presencia de contaminantes en aguas marinas mexicanas: Estero de Punta Banda, Baja California”. Fue financiado por la Estación de Investigación Oceanográfica de Ensenada de la Secretaría de Marina después de ser autorizado por la Dirección General Adjunta de Oceanografía, Hidrografía y Meteorología (DIGAOHM). 


\section{LITERATURA CITADA}

American Ornithologist's Union (AOU). 1998. Check-list of North American birds. 7th edition, Washington, D.C. USA.

Anderson, D. W., F. Gress \& K. F. Mais. 1982. Brown Pelicans: influence of food supply on reproduction. Oikos, 39: 23-31.

Bibby, C. J., N. D. Burgess \& D.A. Hill. 1993. Bird Census Techniques. Academic Press. 257 pp.

Buchsbaum, R. 2003. Some anticipated consequences of global climate change on Massachusetts: Implications for the nature of Massachusetts and ecological management on Massachusetts Audobon Society Sanctuaries: A preliminary Report.

Cairns, D. K. 1987. Seabirds as indicators of marine food supplies. Biological Oceanography, 5: 261271.

Clarke, K. R. \& R. M. Warwick. 2001. Change in Marine Communities: An approach to statistical analysis and interpretation. Plymouth Marine Laboratory, U. K., Segunda Ed., 154 pp.

Cogswell, H. L. 1977. Waterbirds of California. University of California Press. 399 pp.

Escofet, A., D. H. Loya Salinas \& J.I. Arredondo. 1988. El Estero de Punta Banda como hábitat de la avifauna. Ciencias Marinas, 14: 73-100.

ESSA. 2000. Las aves de ESSA. Guía de campo. Exportadora de Sal S. A. de C. V. 201 pp.

Jiménez Pérez L. C. 2003. Mortandad de fulmar norteño (Fulmarus glacialis) en la Bahía de Todos Santos. Tarjeta informativa. Estación de Investigación Oceanográfica. Secretaria de Marina.

Nishikawa, K. K. 1983. Consideraciones sobre el posible impacto ambiental de la instalación de la fábrica de jackets petroleros Bos-Pacific en el Estero de Punta Banda. P.10-20. En Informe preliminar sobre el posible impacto que la empresa Bos-Pacific ocasionará al instalarse en el Estero de Punta Banda, B. C., CICESE. 60 pp.

NOM-059-ECOL. 2001. Norma Oficial Mexicana, Protección ambiental-Especies nativas de México de flora y fauna silvestres. Categorías de riesgo y especificaciones para su inclusión, exclusión o cambio-lista de especies en riesgo. Secretaría del Medio Ambiente y Recursos Naturales. Diario Oficial de la Federación, miércoles 6 de marzo de 2002.

Palacios, E., A. Escofet \& D. H. Loya Salinas. 1991. El Estero de Punta Banda, Baja California, México como eslabón del "Corredor del Pacifico": abundancia de aves playeras. Ciencias Marinas, 17: 109-131.

Periódico Oficial del Estado de Baja California. 1995. En una versión abreviada del Programa de Desarrollo Urbano del Centro de Población de Ensenada, B.C. Sección 1. Tomo CII No.2. 13 de Enero de 1995.

Peterson, R. T. \& E. L. Chalif. 1998. Aves de México, Guía de campo. Editorial Diana, México. 473 pp.

Schaffner, F. C. 1986. Trends in Elegant Tern and Northern Anchovy populations in California. Condor, 88: 347-354.

Springer, A. M., D. G. Roseneau, D. S. Lloyd, C. P. McRoy \& E. C. Murphy. 1986. Seabird responses to fluctuating prey availability in the eastern Bering Sea. Marine Ecology Progress Series, 32: $1-12$.

Valencia, I. D. 2005. Mexico designates four Ramsar sites for World Wetlands Day. The Ramsar Bulletin Board 2 february.

Zar, J. H. 1984. Biostatistical Analysis. Prentice-Hall, England Hills, 672 pp.

Zedler, J. B., C. S. Norby \& B. E. Kus. 1992. The ecology of Tijuana River Estuary, California: A National Resources management Reserve. NOAA, Office of Coastal Resources management Sanctuaries and Reserves Division, Washington, D. C., 150 pp. 


\section{Apéndice 1}

Aves Costeras registradas en el Estero de Punta Banda entre marzo de 2004 y marzo de 2005. At $=$ áreas terrestres adyacentes, $\mathrm{B}=$ Boca del Estero, $\mathrm{Ba}=$ Barra Arenosa, $\mathrm{Ca}=$ Campos Agrícolas, $\mathrm{Cp}=$ Canal principal, $\mathrm{Cs}=$ Canales secundarios, $\mathrm{Cos}=$ Caseríos, $\mathrm{Cv}=$ Caminos Vecinales, $\mathrm{Chm}=$ Charcas de marea, $\mathrm{Dcr}=$ Dique de cantos rodados, $\mathrm{L}=$ Lagunita, $\mathrm{M}=$ Motel, $\mathrm{MA}=$ Marisma Alta, Map = Marisma principal, $\mathrm{Mab}=$ Marisma baja, $\mathrm{Pa}=$ Playa arenosa, $\mathrm{Pm}=$ Planicies de marea, $\mathrm{Tr}=$ Transición, $\mathrm{Tu}=$ Tulares.

Las aves en negritas están listadas en la NOM-059 2001 con los estatus de A= amenazada. $\operatorname{Pr}=$ sujeta a protección especial y el (número de avistamientos).

\begin{tabular}{|c|c|c|c|}
\hline Familia & Nombre científico & Categoría y (N) & Ambiente \\
\hline Gaviidae & Gavia arctica & (3) & $\mathrm{Cp}$ \\
\hline \multirow[t]{2}{*}{ Podicipedidae } & Aechmophorus accidentalis & (7) & $\mathrm{Cp}$ \\
\hline & Podiceps nigricollis & (8) & $\mathrm{L}, \mathrm{Ba}$ \\
\hline \multirow[t]{2}{*}{ Pelecanidae } & Pelecanus erythrorhynchos & (1) & $\mathrm{L}$ \\
\hline & Pelecanus occidentalis & (13) & $\mathrm{Cp}, \mathrm{Ba}$ \\
\hline Phalacrocoracidae & Phalacrocorax auritus & (13) & $\mathrm{Cp}, \mathrm{Ba}$ \\
\hline \multirow[t]{7}{*}{ Ardeidae } & Ardea herodias & (13) & $\mathrm{MA}, \mathrm{Cs}, \mathrm{Chm}$ \\
\hline & Egretta caerulea & (4) & $\mathrm{MA}, \mathrm{Cs}, \mathrm{Chm}$ \\
\hline & Egretta rufescens & $\operatorname{Pr}(5)$ & $\mathrm{MA}, \mathrm{Cs}, \mathrm{Chm}$ \\
\hline & Egretta thula & (13) & $\mathrm{MA}, \mathrm{Cs}, \mathrm{Chm}$ \\
\hline & Ardea alba & (13) & $\mathrm{MA}, \mathrm{Cs}, \mathrm{Chm}$ \\
\hline & Egretta tricolor & (3) & $\mathrm{MA}, \mathrm{Cs}, \mathrm{Chm}$ \\
\hline & Nycticorax nycticorax & (4) & $\mathrm{MA}, \mathrm{Cs}, \mathrm{Chm}$ \\
\hline Threskiornithidae & Plegadis chihi & (3) & $\mathrm{Ca}$ \\
\hline Cathartidae & Cathartes aura & $(8)$ & At \\
\hline \multirow[t]{15}{*}{ Anatidae } & Branta bernicla & $\mathrm{A}(4)$ & $\mathrm{Cp}$ \\
\hline & Anser albifrons & (1) & $\mathrm{Cv}$ \\
\hline & Anas americana & (8) & $\mathrm{L}$ \\
\hline & Anas acuta & (5) & $\mathrm{L}$ \\
\hline & Anas platyrhynchos & (4) & $\mathrm{L}$ \\
\hline & Anas discors & (2) & $\mathrm{L}$ \\
\hline & Anas cyanoptera & (5) & $\mathrm{L}$ \\
\hline & Anas clypeata & (4) & $\mathrm{L}$ \\
\hline & Aythya americana & $(5)$ & $\mathrm{L}$ \\
\hline & Aythya affinis & (4) & $\mathrm{L}$ \\
\hline & Bucephala albeola & (7) & $\mathrm{Cp}$ \\
\hline & Melanitta perspicillata & (7) & $\mathrm{Cp}$ \\
\hline & Melanitta fusca & (2) & $\mathrm{Cp}$ \\
\hline & Mergus serrator & (4) & $\mathrm{Cp}$ \\
\hline & Mergus merganser & (1) & $\mathrm{Cp}$ \\
\hline
\end{tabular}




\begin{tabular}{|c|c|c|c|}
\hline Familia & Nombre científico & Categoría y (N) & Ambiente \\
\hline \multirow[t]{6}{*}{ Accipitridae } & Pandion haliaetus & (13) & MA, Cp \\
\hline & Circus cyaneus & $(6)$ & $\mathrm{MA}, \mathrm{Cv}$ \\
\hline & Elanus leucurus & (3) & $\mathrm{Cv}$ \\
\hline & Buteo swainsoni & $\operatorname{Pr}(1)$ & $\mathrm{Cv}$ \\
\hline & Buteo jamaiciensis & (1) & $\mathrm{Cv}$ \\
\hline & Aquila chrysaetos & $\mathrm{A}(2)$ & $\mathrm{Ba}$ \\
\hline \multirow[t]{3}{*}{ Falconidae } & Falco sparverius & (6) & $\mathrm{Cv}$ \\
\hline & Falco peregrinus & $\operatorname{Pr}(3)$ & $\mathrm{Cv}$ \\
\hline & Falco mexicanus & $A(2)$ & $\mathrm{Cv}$ \\
\hline Odontophoridae & Callipepla californica & (6) & $\mathrm{Ba}$ \\
\hline \multirow[t]{2}{*}{ Rallidae } & Rallus longirostris & $\operatorname{Pr}(9)$ & Mab \\
\hline & Fulica americana & $(10)$ & $\mathrm{L}$ \\
\hline \multirow[t]{4}{*}{ Charadriidae } & Charadrius semipalmatus & (9) & $\mathrm{Pm}$ \\
\hline & Charadrius vociferus & (5) & M \\
\hline & Charadrius alexandrinus & (2) & $\mathrm{Ba}$ \\
\hline & Pluvialis squatarola & (5) & $\mathrm{Pm}$ \\
\hline \multirow[t]{2}{*}{ Recurvirostridae } & Recurvirostra americana & (8) & $\mathrm{L}$ \\
\hline & Himantopus mexicanus & (13) & $\mathrm{L}$ \\
\hline \multirow[t]{17}{*}{ Scolopacidae } & Limosa fedoa & (13) & $\mathrm{Pm}$ \\
\hline & Catoptrophorus semipalmatus & (13) & $\mathrm{Pm}$ \\
\hline & Tringa solitaria & (4) & $\mathrm{Pm}$ \\
\hline & Tringa flavipes & (3) & $\mathrm{Pm}$ \\
\hline & Tringa melanoleuca & (5) & Pm \\
\hline & Limnodromus griseus & (9) & $\mathrm{Pm}$ \\
\hline & Numenius americanus & (13) & $\mathrm{Pm}$ \\
\hline & Numenius phaeopus & $(5)$ & $\mathrm{Pm}$ \\
\hline & Arenaria interpres & (2) & Dcr \\
\hline & Arenaria melanocephala & (1) & Dcr \\
\hline & Calidris alpina & (1) & Dcr \\
\hline & Calidris canutus & (1) & Dcr \\
\hline & Calidris minutilla & (7) & $\mathrm{Pm}$ \\
\hline & Calidris mauri & (3) & $\mathrm{Pm}$ \\
\hline & Calidris alba & (1) & $\mathrm{Pa}$ \\
\hline & Phalaropus fulicaria & (2) & $\mathrm{L}$ \\
\hline & Phalaropus lobatus & (3) & $\mathrm{L}$ \\
\hline \multirow[t]{5}{*}{ Lariidae } & Larus occidentalis & (13) & $\mathrm{L}, \mathrm{Ba}$ \\
\hline & Larus heermanni & $\operatorname{Pr}(2)$ & B \\
\hline & Larus delawarensis & (1) & $\mathrm{L}$ \\
\hline & Sterna forsteri & (8) & $\mathrm{Cp}, \mathrm{L}$ \\
\hline & Sterna caspia & (3) & $\mathrm{Cp}, \mathrm{L}$ \\
\hline
\end{tabular}




\begin{tabular}{|c|c|c|c|}
\hline Familia & Nombre científico & Categoría y (N) & Ambiente \\
\hline \multirow[t]{5}{*}{ Lariidae } & Sterna antillarum & $\operatorname{Pr}(6)$ & $\mathrm{Cp}, \mathrm{L}$ \\
\hline & Sterna hirundo & $(6)$ & $\mathrm{Cp}, \mathrm{L}$ \\
\hline & Sterna maxima & (5) & $\mathrm{Cp}, \mathrm{L}$ \\
\hline & Sterna elegans & $\operatorname{Pr}(7)$ & $\mathrm{Cp}, \mathrm{L}$ \\
\hline & Rynchops niger & $(13)$ & Pm, L \\
\hline \multirow[t]{2}{*}{ Columbidae } & Zenaida macroura & (13) & $\mathrm{Cv}$ \\
\hline & Columba livia & (13) & $\operatorname{Cos}$ \\
\hline Cucullidae & Geococcyx californianus & (2) & $\mathrm{Ba}$ \\
\hline \multirow[t]{2}{*}{ Stringidae } & Asio flammeus & $\operatorname{Pr}(3)$ & $\mathrm{Ba}$ \\
\hline & Athene cunicularia & (2) & $\mathrm{Ba}$ \\
\hline Trochilidae & Calypte costae & (1) & $\operatorname{Tr}$ \\
\hline Alcedinidae & Ceryle alcion & (7) & $\mathrm{L}, \mathrm{B}$ \\
\hline \multirow{5}{*}{ Tirannidae } & Sayornis nigricans & (7) & $\mathrm{Cv}$ \\
\hline & Sayornis saya & (3) & $\operatorname{Tr}$ \\
\hline & Pyrocephalus rubinus & (2) & $\operatorname{Tr}$ \\
\hline & Tyrannus vociferans & (2) & $\operatorname{Tr}$ \\
\hline & Tyrannus verticalis & (1) & $\operatorname{Tr}$ \\
\hline Laniidae & Lanius ludovicianus & (1) & $\operatorname{Tr}$ \\
\hline Corvidae & Corvus corax & (9) & At \\
\hline \multirow[t]{3}{*}{ Hirundinidae } & Petrochelidon pyrrhonota & (5) & $\mathrm{Ba}, \mathrm{Pm}$ \\
\hline & Petrochelidon fulva & (1) & $\mathrm{Ba}, \mathrm{Pm}$ \\
\hline & Hirundo rustica & (2) & $\mathrm{Ba}, \mathrm{Pm}$ \\
\hline Turdidae & Myadestes townsendi & (1) & $\operatorname{Tr}$ \\
\hline Mimidae & Mimus polyglottos & (13) & $\operatorname{Tr}$ \\
\hline Sturnidae & Sturnus vulgaris & $(9)$ & $\mathrm{Cv}$ \\
\hline \multirow[t]{2}{*}{ Emberizidae } & Passerculus sandwichensis & $\mathrm{A}(8)$ & MA \\
\hline & Zonotrichia leucophrys & (13) & $\mathrm{Cv}$ \\
\hline Cardinalidae & Guiraca caeruea & $(1)$ & $\mathrm{Ca}$ \\
\hline \multirow[t]{5}{*}{ Icteridae } & Agelaius phoeniceus & (3) & $\mathrm{Tu}$ \\
\hline & Sturnella neglecta & $(5)$ & $\operatorname{Tr}$ \\
\hline & Euphagus cyanocephalus & (13) & $\operatorname{Tr}$ \\
\hline & Molothus ater & $(1)$ & $\mathrm{Ca}$ \\
\hline & Icterus cucullatus & $(2)$ & $\mathrm{Ca}$ \\
\hline Fringillidae & Carpodacus mexicanus & (13) & $\operatorname{Cos}$ \\
\hline
\end{tabular}

